\title{
On the coordinate rings of quiver varieties associated to extended Dynkin diagrams
}

\author{
By \\ Yasuhiro OMODA
}

\section{Introduction}

P. B. Kronheimer $[\mathrm{Kr}]$ has constructed quiver varieties from Extended Dynkin Diagrams of types of $\tilde{A}_{n}, \tilde{D}_{n}, \tilde{E}_{n}$. These quiver varieties are important objects for the study of simple singularities. Let $p$ be the quotient map of Cartan subalgebra by Weyl group. The semiuniversal deformations of simple singularities are constructed on the quotient space of Cartan subalgebra by Weyl group of corresponding types. Then these quiver varieties are the pull-back of semiuniversal deformations of simple singularities by the quotient map $p$. These quiver varieties are obtained as the symplectic quotients of symplectic vector spaces by a reductive group. So the coordinate rings are invariant subrings of polynomial rings with respect to the action of the group. In general it is difficult to find a minimal set of generators of an invariant ring and the relations between them. In this paper we show that it is possible for the case of quiver varieties which were constructed by P. B. Kronheimer. Moreover surprisingly we can show that the obtained relation is unique and irreducible. In this research we used the invariant theory of quivers by Le Bruyn, and Procesi, $\mathrm{C}[\mathrm{BP}]$ and of matrices of low degrees by K. Nakamoto [Na 1,2].

The author wishes to express his hearty thanks to A. Ishii, A. Kono, J. Matsuzawa, K. Nakamoto, I. Naruki, and K. Saito with whom he had valuable discussions.

\section{Preliminary $|\mathbf{S} 2|$}

2.1. Simple singularities $[\mathbf{S} 1,2]$. Let $\Gamma$ be a finite subgroup of $S U(2) . \quad \Gamma$ is isomorphic to one of following groups:

$$
\begin{array}{cl}
C_{n} & \text { cyclic group of order } n, \\
D_{n} & \text { binary dihedral group of order } 4 n, \\
T & \text { binary tetrahedral group, } \\
O & \text { binary octahedral group, } \\
I & \text { binary icosahedral group. }
\end{array}
$$


We call a singularity which $\mathbf{C}^{2} / \Gamma$ have at 0 simple singularity. For $S=\mathbf{C}^{2} / \Gamma$, we write a minimal resolution of $S$ by

$$
\pi: \tilde{S} \rightarrow S .
$$

Hence the exceptional fibre $\pi^{-1}(0)$ is:

$$
\pi^{-1}(0)=C_{1} \cup \cdots \cup C_{n} .
$$

Here each $C_{i}$ is $\boldsymbol{P}^{1}$ and intersection matrix $C:=\left(C_{i} \cdot C_{j}\right)$ is Cartan matrix of following type $\triangle(\Gamma)$.

\begin{tabular}{c|c|c|c|c|c}
$\Gamma$ & $C_{n}$ & $D_{n}$ & $T$ & $O$ & $I$ \\
\hline$\triangle(\Gamma)$ & $A_{n-1}$ & $D_{n+2}$ & $E_{6}$ & $E_{7}$ & $E_{8}$
\end{tabular}

By this correspondence we call $\mathbf{C}^{2} / \Gamma$ simple singularity of type $\triangleq(\Gamma)$.

Let $\mathbf{C}^{\times}$be multiplicative group. We define the action of $\mathbf{C}^{\times}$with weight $\left(w_{1}, \ldots, w_{n}\right)$ on $V=\mathbf{C}^{n}$ :

$$
\begin{aligned}
& t \cdot\left(x_{1}, \ldots, x_{n}\right)=\left(t^{m 1} x_{1}, \ldots, t^{n n} x_{n}\right) \\
& \quad \text { for }\left(x_{1}, \ldots, x_{n}\right) \in V, \quad t \in \mathbf{C}^{\times} .
\end{aligned}
$$

Moreover when $\mathbf{C}^{\times}$acts on $W=\mathbf{C}^{m}$ with weight $\left(d_{1}, \ldots, d_{m}\right)$ and

$$
f: V \rightarrow W
$$

is $\mathbf{C}^{\times}$-equivariant map, we say that $f$ is a quasi-homogeneous map of weight $\left(d_{1}, \ldots d_{m} ; w_{1}, \ldots, w_{n}\right)$. Next proposition is well known.

Proposition 1. Assume that $f \in \mathbf{C}[X, Y, Z]$ is a quasi-homogeneous polynomial of a weight $\left(d ; w_{1}, w_{2}, w_{3}\right)$ in the next list. Hence if Spec $\mathbf{C}[X, Y, Z] /(f)$ have an isolated singularity, $f=0$ represents a simple singularity of the corresponding type.

\begin{tabular}{c|c|c|c|c} 
& $d$ & $w_{1}$ & $w_{2}$ & $w_{3}$ \\
\hline$A_{n}$ & $2(n+1)$ & 2 & $n+1$ & $n+1$ \\
\hline$D_{n}$ & $4(n-1)$ & 4 & $2(n-2)$ & $2(n-1)$ \\
\hline$E_{6}$ & 24 & 6 & 8 & 12 \\
\hline$E_{7}$ & 36 & 8 & 12 & 18 \\
\hline$E_{8}$ & 60 & 12 & 20 & 30
\end{tabular}

\subsection{Quiver $[\mathrm{S} 2]$}

Definition 1. We call two finite sets $A, V$ with two maps $h, t$ from $A$ to $V$ quiver. i.e. It is just an oriented finite graph.

Example 1. $1 \bullet \rightarrow \bullet 2$

In this case $A$ has only one element $\{a\}, V$ is $\{1,2\}, h(a)=2$ and $t(a)=1$. 
When a quiver is given, we fix $\alpha=\left(\alpha_{i} ; i \in V, \alpha_{i} \in \mathbf{N}\right)$ and put $V_{i}=\mathbf{C}^{\alpha_{i}}$. We introduce following notation:

$$
\begin{gathered}
\mathfrak{R}(V, A, \alpha):=\bigoplus_{a \in A} \operatorname{Hom}_{\mathbf{C}}\left(V_{t(a)}, V_{h(a)}\right), \\
G L(V, A, \alpha):=\Pi_{i \in V} G L\left(V_{i}\right) .
\end{gathered}
$$

We define an action of $G L(V, A, \alpha)$ on $\mathfrak{R}(V, A, \alpha)$ by following way:

$$
\begin{gathered}
g \cdot\left\{f_{a}\right\}:=\left\{g_{h(a)} f_{a} g_{t(a)}^{-1}\right\}, \\
\text { for } g=\left\{g_{i}\right\} \in G L(V, A, \alpha),\left\{f_{a}\right\} \in \mathfrak{R}(V, A, \alpha) .
\end{gathered}
$$

For $\left\{f_{a}\right\} \in \mathfrak{R}(V, A, \alpha)$ and $\left(a_{1}, a_{2}, \ldots, a_{s}\right) \in A \times \cdots \times A$, if $h\left(a_{1}\right)=t\left(a_{2}\right), \ldots$, $h\left(a_{s-1}\right)=t\left(a_{s}\right)$, we can consider the map:

$$
f_{a_{s}} \circ f_{a_{s-1}} \circ \cdots \circ f_{a_{1}}: V_{t\left(a_{1}\right)} \rightarrow V_{h\left(a_{s}\right)} .
$$

Moreover if $t\left(a_{1}\right)=h\left(a_{s}\right)$, we call the composed map cycle. Hence following theorem is proved by $[\mathrm{BP}]$ :

Theorem 1. The invariant ring $\mathbf{C}[\mathfrak{R}(V, A, \alpha)]^{G L(V, A, \alpha)}$ is generated by the functions which give the traces of cycles.

2.3. Quiver varieties $[\mathbf{N} \mid$. Suppose a finite graph is given. We assume that no edge may join a vertex with itself. Let $A$ be the set of pairs consisting of an edge together with an orientation of it. Let $t(a)$ (resp. $h(a))$ be the outgoing (resp. incoming) vertex of $a \in A$. For $a \in A$ we denote by $\bar{a}$ the same edge as $a$ with the reverse orientation. Moreover let $V$ be the set of vertexes of the graph. Hence we obtained a quiver. Choose a subset $\Omega \subset A$ such that $\bar{\Omega} \cup \Omega=$ $A, \bar{\Omega} \cap \Omega=\varnothing$. Such a choice of the subset is called an orientation of the graph. The choice of the orientation is not essential. Our constructions are essentially independent of $\Omega$. From now on we consider only such a quiver with an orientation. Let $V$ be $\{0,1, \ldots, n\}$. Let $T \subset G L(V, A, \alpha)$ be scalar. Hence $T$ acts trivially on $\mathfrak{R}(V, A, \alpha)$. Let $P G L(V, A, \alpha)$ be $G L(V, A, \alpha) / T, \operatorname{Lie}\{P G L(V . A, \alpha)\}$ Lie algebra of $P G L(V, A, \alpha)$ and $(\operatorname{Lie}\{P G L(V, A, \alpha)\})^{\star}$ the dual space of Lie algebra $\operatorname{Lie}\{P G L(V, A, \alpha)\}$. Then $P G L(V, A, \alpha)$-invariant holomorpic symplectic form (.) and corresponding moment map $\mu_{\mathbf{C}}$ are given as follows:

$$
\begin{aligned}
\left(\left\{f_{a}\right\},\left\{g_{a}\right\}\right)= & \sum_{a \in A} \varepsilon(a) \operatorname{tr}\left(f_{a} g_{a}\right) \\
\mu_{\mathbf{C}}: & \mathfrak{R}(V, A, \alpha) \rightarrow(\operatorname{Lie}\{P G L(V, A, \alpha)\})^{\star} \subset \oplus_{i \in V} M_{x_{i}} \\
& \mu_{\mathbf{C}}\left(\left\{f_{a}\right\}\right)=\bigoplus_{i \in V}\left(-\sum_{a \in A, h(a)=i} \varepsilon(a) f_{a} f_{\bar{a}}\right) \\
\text { for }\left\{f_{a}\right\}, & \left\{g_{a}\right\} \in \mathfrak{R}(V, A, \alpha)
\end{aligned}
$$

where $\varepsilon(a)=1$ if $a \in \Omega, \varepsilon(a)=-1$ if $a \in \bar{\Omega}$. Let $Z$ be the dual space of the centre of $\operatorname{Lie}\{P G L(V, A . \alpha)\}$. Then we define the Quiver variety for $\zeta_{\mathbf{C}} \in Z$ : 


$$
\mathscr{H}_{\zeta_{\mathrm{C}}}:=\mu_{\mathrm{C}}^{-1}\left(\zeta_{\mathbf{C}}\right) / / P G L(V, A, \alpha) .
$$

Here $\mu_{\mathbf{C}}^{-1}\left(\zeta_{\mathbf{C}}\right) / / P G L(V, A, \alpha)$ is categorical quotient.

Remark 1. Note $\mathscr{U}_{0}$ and $\mu_{\mathbf{C}}^{-1}(Z) / / P G L(V, A, \alpha)$ are essentially independent of a orientation of the graph.

We define the action of $\mathbf{C}^{\times}$on $\mathfrak{R}(V, A, \alpha)$ by scalar action. Then this action preserves $\mu_{\mathbf{C}}^{-1}(Z)$, and $\mu_{\mathbf{C}}^{-1}(0)$. Moreover this action commutes with the action of $G L(V, \boldsymbol{A}, \alpha)$. So we may consider $\mathbf{C}\left[\mu_{\mathbf{C}}^{-1}(Z)\right]^{G L(V, A, \alpha)}$ and $\mathbf{C}\left[\mu_{\mathbf{C}}^{-1}(0)\right]^{G L(V, A, \alpha)}$ as graded rings.

Definition 2. For a given graph we put $d_{i j}=1$ if vertex $i$ is joined to $j$, and $d_{i j}=0$ if vertex $i$ isn't jointed to $j$ and $d_{i i}=-2$. We define $D:=\left(d_{i j}\right)$.

We have:

Proposition 2. $\operatorname{dim} \mu_{\mathbf{C}}^{-1}(Z) / / P G L(V, A, \alpha) \geq n+2-{ }^{t} \alpha D \alpha$.

Proof.

$$
\begin{aligned}
\operatorname{dim} \mu_{\mathbf{C}}^{-1}(Z) / / P G L(V, A, \alpha) & \geq \operatorname{dim} \mu_{\mathbf{C}}^{-1}(Z)-\operatorname{dim} P G L(V, A, \alpha) \\
& \geq \operatorname{dim} \mathfrak{R}(V, A, \alpha)-2 \operatorname{dim} P G L(V, A, \alpha)+\operatorname{dim} Z \\
& =n+2-{ }^{t} \alpha D \alpha
\end{aligned}
$$

2.4. McKay's observation [M]. Let $\Gamma$ be a finite subgroup of $S U(2)$. Let $R_{0}, R_{1}, \ldots, R_{n}$ be irreducible representations of $\Gamma$. Here $R_{0}$ is the trivial representation. Let $Q$ be the natural 2-dimensional representation which is obtained from a inclusion: $\Gamma \subset S U(2)$. We put for $i, j=0,1, \ldots, n$ :

$$
\begin{gathered}
n_{i}:=\operatorname{dim}_{\mathbf{C}} R_{i} \\
m_{i j}:=\operatorname{dim}_{\mathbf{C}} \operatorname{Hom}_{\Gamma}\left(R_{i}, R_{j} \otimes Q\right) \\
c_{i j}:=2 \delta_{i j}-m_{i j} \\
(i, j=0,1, \ldots, n)
\end{gathered}
$$

Hence Mckay observed following fact.

Theorem 2. $C=\left(c_{i j}\right)$ is a Cartan matrix associated to Extended Dynkin diagram of type $\tilde{\triangle}(\Gamma)$.

Let $R$ be the regular representation of $\Gamma$.

$$
\begin{aligned}
(\operatorname{End}(R) \otimes Q)^{\Gamma} & =\operatorname{Hom}_{\Gamma}(R, R \otimes Q) \\
& =\operatorname{Hom}_{\Gamma}\left(\sum_{i=0}^{n} \mathbf{C}^{n_{i}} \otimes R_{i},\left(\sum_{j=0}^{n} \mathbf{C}^{n_{j}} \otimes R_{j}\right) \otimes Q\right) \\
& =\bigoplus_{i, j}\left\{\operatorname{Hom}\left(\mathbf{C}^{n_{i}}, \mathbf{C}^{n_{j}}\right) \otimes \mathbf{C}^{m_{i j}}\right\}
\end{aligned}
$$


Hence above theorem says that $(\operatorname{End}(R) \otimes Q)^{\Gamma}$ is $\mathfrak{R}(V, A, \alpha)$ which is obtained from following quiver of type $\tilde{\triangle}(\Gamma)$ and $\alpha$. We call following quivers McKay quivers.
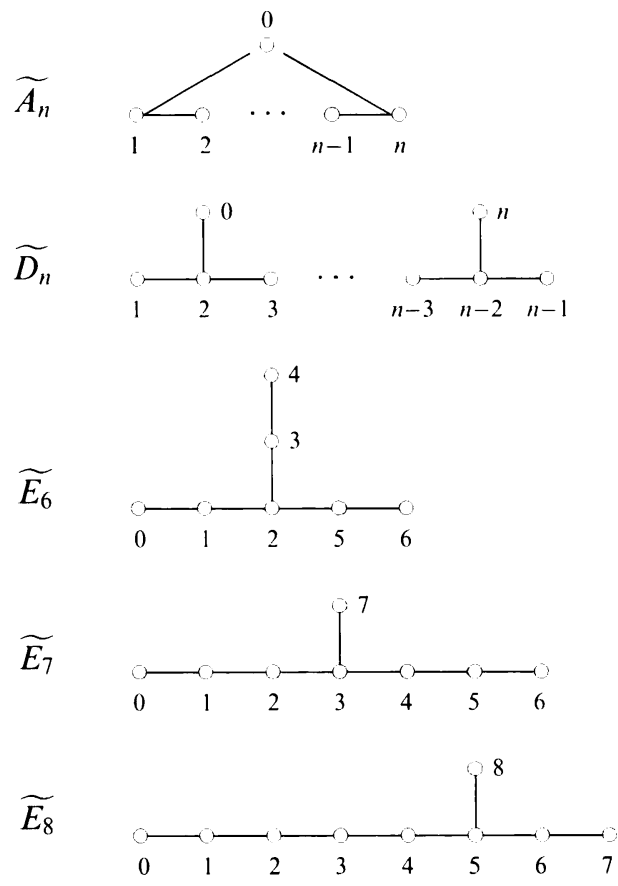

$$
\begin{aligned}
& V=\{0,1, \ldots, n\} \\
& \alpha=(1, \ldots, 1) \\
& V=\{0,1, \ldots, n\} \\
& \alpha=(1,1,2,2, \ldots, 2,1,1)
\end{aligned}
$$

$V=\{0,1,2,3,4,5,6\}$

$\alpha=(1,2,3,2,1,2,1)$

$V=\{0,1,2,3,4,5,6,7\}$

$\alpha=(1,2,3,4,3,2,1,2)$

$V=\{0,1,2,3,4,5,6,7,8\}$

$\alpha=(1.2,3,4,5,6,4,2,3)$

Here the numbering on diagrams indicate vertexes.

2.5. Hyperkahler quotient and simple singularities $[\mathbf{K r}]$. Natural representation $Q$ of $\Gamma$ has a $\Gamma$-invariant quaternionic structure. We equip regular representation $R$ of $\Gamma$ with a $\Gamma$-invariant Hermitian inner product. Let $(\operatorname{End}(R))^{h}$ be the set of all Hermitian maps of $\operatorname{End}(R)$. Thus following module has $\Gamma$ invariant right quaternionic structure.

$$
M:=\operatorname{End}(R) \otimes_{\mathbf{C}} Q \cong(\operatorname{End}(R))^{h} \otimes_{\mathbf{R}} \mathbf{H}
$$

Here $\mathbf{H}$ is quaternion. Let $U(R)$ denote the unitary group acting on $\operatorname{End}(R)$ by conjugation, and let

$$
\begin{aligned}
U(\Gamma) & :=\left\{g \in U(R) \mid g \gamma=\gamma g \quad{ }^{\forall} \gamma \in \Gamma\right\} \\
S U(\Gamma) & :=U(\Gamma) \cap S L(R)
\end{aligned}
$$

Because of $R \cong \bigoplus_{i}\left\{R_{i} \otimes \mathbf{C}^{n_{i}}\right\}$ we have

$$
\begin{aligned}
U(\Gamma) & \cong \prod_{i} U\left(n_{i}\right) \\
S U(\Gamma) & \cong\left\{\left(A_{0}, \ldots, A_{n}\right) \in U(\Gamma) \mid \prod_{i} \operatorname{det}\left(A_{i}\right)=1\right\}
\end{aligned}
$$


The compact group $U(\Gamma)$ acts on $M$ and on

$$
M(\Gamma):=(\operatorname{End}(R) \otimes Q)^{\Gamma}
$$

preserving the quaternionic structure. We obtain a $U(I)$-equivariant hyper-kähler moment map

$$
\mu_{\mathbf{H}}: M(\Gamma) \rightarrow \underline{u}(\Gamma)^{\star} \otimes_{\mathbf{R}} \mathbf{H}_{0}
$$

where $\mathbf{H}_{0}=\mathbf{R} I \oplus \mathbf{R} J \oplus \mathbf{R} K$ denotes the pure quaternions, and where $u(\Gamma)^{\star}$ is the dual of the Lie algebra $u(\Gamma)$ of $U(\Gamma)$ acted on by $U(\Gamma)$ via the coadjoint action. Since the diagonal scalars:

$$
\mathbf{T}=\left\{(\lambda, \ldots \lambda) \in \prod_{i} U\left(\alpha_{i}\right) \mid \lambda \in U(1)\right\}
$$

act trivially on $M(\Gamma)$ and since $u(\Gamma)^{\star}$ may be identified with $u(\Gamma)$ by means of the trace form $(A, B) \mapsto \operatorname{tr}\left(A B^{\star}\right)$, the target of $\mu_{\mathbf{H}}$ may be identified with $s u(\Gamma) \otimes_{\mathbf{R}} \mathbf{H}_{0}$, where $s u(\Gamma)$ is the Lie algebra of $S U(\Gamma)$. We put

$$
\mathbf{c}=\left\{\left(\mu_{0}, \ldots, \mu_{n}\right) \in \bigoplus_{i} u\left(\alpha_{i}\right) \mid \mu_{i} \in u(1) \sum_{i} \alpha_{i} \mu_{i}=0\right\} .
$$

Then $\mathbf{c}$ is the $r$-dimensional centre of $s u(\Gamma)$, i.e.

$$
\mathbf{c}=(\operatorname{su}(\Gamma))^{U(\Gamma)} \text {. }
$$

Then $U(\Gamma)$ acts on a fibre $\mu_{\mathbf{H}}^{-1}(\zeta), \zeta=\zeta_{1} I+\zeta_{2} J+\zeta_{3} K \in \mathbf{c} \otimes \mathbf{H}_{0}$, and the real differential geometric quotient $\mu_{\mathbf{H}}^{-1}(\zeta) / U(\Gamma)$ is a hyper-kahler quotient (at least at its smooth points it carries the structure of a hyper-kahler manifold). Kronheimer showed that for all $\zeta \in \mathbf{c} \otimes \mathbf{H}_{0}$, the quotient $\mu_{\mathbf{H}}^{-1}(\zeta) / U(\Gamma)$ is a complex-analytic surface with at most isolated (simple) singularities, in particular

$$
\mu_{\mathbf{H}}^{-1}(0) / U(\Gamma) \cong \mathbf{C}^{2} / \Gamma \text {. }
$$

Moreover, there is a natural identification of $\mathbf{c} \otimes \mathbf{C}=\mathbf{c} \otimes \boldsymbol{J} \otimes \mathbf{c} \otimes \boldsymbol{K}$ with a Cartan subalgebra $\mathbf{h}$ of type $\underline{\Delta}(\Gamma)$ such that the complex $r$-parameter family

$$
\mu_{\mathbf{H}}^{-1}(\mathbf{c} \otimes \mathbf{C}) / U(\Gamma) \rightarrow \mathbf{c} \otimes \mathbf{C}
$$

realizes a pull-back (via $\mathbf{h} \rightarrow \mathbf{h} / W$ ) of the semiuniversal deformation of $\mathbf{C}^{2} / \Gamma$, and shifting this family into the $I$-direction provides a simultaneous resolution

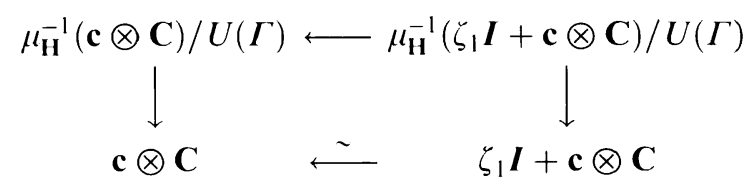

(for generic $\zeta_{1} \in \mathbf{c}$ )

2.6. The invariant theory of $(n, n)$-matrices $(n=2,3)$. We denote the set of all $(n . n)$-matrices by $M_{n}$. We define $G L(n)$-action on $M_{n} \times \cdots \times M_{n}$ by 


$$
\begin{gathered}
\quad g\left(x_{1}, \ldots, x_{s}\right):=\left(g x_{1} g^{-1}, \ldots, g x_{s} g^{-1}\right), \\
\text { for } g \in G L(n), \quad\left(x_{1}, \ldots, x_{s}\right) \in M_{n} \times \cdots \times M_{n} .
\end{gathered}
$$

We denote the coordinate of $M_{n} \times M_{n} \times M_{n}$ by $(A, B, C)$ in the form of $(n, n)$ matrices and the coordinate of $M_{n} \times M_{n}$ by $(A, B)$ in the form of $(n, n)$-matrices. Hence next theorems are proved in [Na1], [Na2].

Theorem 3. $\mathrm{C}\left[M_{2} \times M_{2} \times M_{2}\right]^{G L(2)}$ is generated by $\{\operatorname{det} A, \operatorname{det} B, \operatorname{det} C, \operatorname{tr} A$, $\operatorname{tr} B, \operatorname{tr} C, \operatorname{tr}(A B), \operatorname{tr}(B C), \operatorname{tr}(C A), \operatorname{tr}(A B C)\}$ and there is a unique relation between these generators as follows,

$$
\begin{aligned}
& \{\operatorname{tr}(A B C)\}^{2}-P_{2}\{\operatorname{tr}(A B C)\}+Q_{2}=0, \\
& \text { where } P_{2}=\operatorname{tr} A \operatorname{tr}(B C)+\operatorname{tr} B \operatorname{tr}(C A)+\operatorname{tr} C \operatorname{tr}(A B)+\operatorname{tr} A \operatorname{tr} B \operatorname{tr} C \text {, } \\
& Q_{2}=\operatorname{det} B \operatorname{det} C(\operatorname{tr} A)^{2}+\operatorname{det} C \operatorname{det} A(\operatorname{tr} B)^{2}+\operatorname{det} A \operatorname{det} B(\operatorname{tr} C)^{2} \\
& +\operatorname{det} C\{\operatorname{tr}(A B)\}^{2}+\operatorname{det} A\{\operatorname{tr}(B C)\}^{2}+\operatorname{det} B\{\operatorname{tr}(C A)\}^{2} \\
& -\operatorname{det} C \operatorname{tr} A \operatorname{tr} B \operatorname{tr}(A B)-\operatorname{det} A \operatorname{tr} B \operatorname{tr} C \operatorname{tr}(B C) \\
& -\operatorname{detBtrCtrAtr}(C A)-4 \operatorname{det} A \operatorname{det} B \operatorname{det} C \\
& +\operatorname{tr}(A B) \operatorname{tr}(B C) \operatorname{tr}(C A) \text {. }
\end{aligned}
$$

Theorem 4. $\mathbf{C}\left[M_{3} \times M_{3}\right]^{G L(3)}$ is generated by $\{\operatorname{det} A, \operatorname{det} B, \operatorname{tr} A, \operatorname{tr} B, \operatorname{tr}(A B)$, $\left.\operatorname{tr}\left(A^{2}\right), \operatorname{tr}\left(B^{2}\right), \operatorname{tr}\left(A^{2} B\right), \operatorname{tr}\left(A B^{2}\right), \operatorname{tr}(A B A B), \operatorname{tr}\left(A B A^{2} B^{2}\right)\right\}$ and there is a unique relation between these generators as follows,

$$
\left\{\operatorname{tr}\left(A B A^{2} B^{2}\right)\right\}^{2}-P_{3}\left\{\operatorname{tr}\left(A B A^{2} B^{2}\right)\right\}+Q_{3}=0 .
$$

Here $P_{3}, Q_{3}$ are polynomials of $\left\{\operatorname{det} A, \operatorname{det} B, \operatorname{tr} A, \operatorname{tr} B, \operatorname{tr}(A B), \operatorname{tr}\left(A^{2}\right), \operatorname{tr}\left(B^{2}\right), \operatorname{tr}\left(A^{2} B\right)\right.$, $\left.\operatorname{tr}\left(A B^{2}\right), \operatorname{tr}(A B A B)\right\}$.

See [Na2] for detail.

3. The structure of $\mathbf{C}\left[\mu_{\mathbf{C}}^{-1}(Z)\right]^{G L(V, A, x)}$ or $\mathbf{C}\left[\mu_{\mathbf{C}}^{-1}(0)\right]^{G L(I ; A, x)}$

We recall the definition of $Z$, i.e. the dual space of the centre of Lie $\{P G L(V, A, \alpha)\}$. In this section we determine a minimal set of generators of $\mathrm{C}\left[\mu_{\mathbf{C}}^{-1}(Z)\right]^{G L(V, A, x)}$. By theorem 1 we may consider only the traces of cycles as generators of these rings. Then these generators are homogeneous elements.

Definition 3. Let $f, g$ are homogeneous elements of $\mathbf{C}\left[\mu_{\mathbf{C}}^{-1}(Z)\right]^{G L(V, A, \alpha)}$ with degree $k$. We say $f$ is equivalent to $g$ when $f-g$ is contained in the ring which is generated by all homogeneous elements with degree $l(<k)$ in $\mathbf{C}\left[\mu_{\mathbf{C}}^{-1}(Z)\right]^{G L(V, A, \alpha)}$ and we write $f \sim g$.

When we By $A_{i, j}$ we denote the coordinate of $\operatorname{Hom}\left(V_{t(a)}, V_{h(a)}\right)$ in $\left(\alpha_{i}, \alpha_{j}\right)$ matrix form for any $a=(i \bullet \rightarrow \bullet j)$. We denote a unit matrix in $M_{n}$ by $E_{n}$. We 
only show the proof of the case of type $\tilde{D}_{n}$ in detail. Since the proof of other cases is similar as type $\tilde{D}_{n}$, we will describe only outline.

3.1. type $\tilde{A}_{n}$. Since $G L(V, A, \alpha)=\left(\mathbf{C}^{\times}\right)^{n+1}$, we have $\mu_{\mathbf{C}}^{-1}(Z)=\mathfrak{R}(V, A, \alpha)$. The action of an element $t=\left(t_{0}, \ldots, t_{n}\right) \in\left(\mathbf{C}^{\times}\right)^{n+1}$ on $\mathfrak{R}(V, A, \alpha)$ is given by $t \cdot A_{i, j}$ $=t_{j} t_{i}^{-1} A_{i, j}$. We have fundamental invariants: $z_{i}=A_{i, j} A_{j, i}(j=i+1 \bmod n+1)$, $x=A_{0,1} \ldots A_{n-1, n} A_{n, 0}, y=A_{0, n} \ldots A_{n-1, n} A_{n, 0}$. The relation of these invariants is only $x y=z_{0} \ldots z_{n}$. The weight of each invariant $\left\{z_{i}, x, y\right\}$ is $\{2, n+1, n+1\}$, and the relation is quasi homogeneous polynomial with weight $(2(n+1) ; 2, \ldots, 2, n+1$, $n+1)$. In paticular $\mathbf{C}\left[\mu_{\mathbf{C}}^{-1}(0)\right]^{G L(\boldsymbol{V}, A, x)}=\mathbf{C}[x, y, z] /\left(x y=z^{n+1}\right)$. It is simple singularity of type $A_{n}$.

\section{2. type $\tilde{D}_{n}(n \geq 4)$}

Theorem 5. For Mckay quiver of type $\tilde{D}_{n} \mathbf{C}\left[\mu_{\mathbf{C}}^{-1}(Z)\right]^{G L(V, A, x)}$ is generated by $(n+3)$ generators and there is a unique irreducible relation between these generators. Moreover this relation is a quasihomogeneous polynomial with weight $(4(n-1)$; $2, \ldots, 2,4,2(n-2), 2(n-1))$. In particular $\mathbf{C}\left[\mu_{\mathbf{C}}^{-1}(0)\right]^{G L(V, A, x)}$ is generated by 3 generators and the relation between these generators represents simple singularity of type $D_{n}$. We write down generators;

$$
\begin{aligned}
& a_{1}=\operatorname{tr}\left(A_{2,0} A_{0,2}\right), \\
& a_{2}=\operatorname{tr}\left(A_{2, \mid} A_{1,2}\right), \\
& a_{3}=\operatorname{tr}\left(A_{3,2} A_{2,3}\right), \\
& \vdots \\
& a_{n-2}=\operatorname{tr}\left(A_{n-2, n-3} A_{n-3, n-2}\right), \\
& a_{n-1}=\operatorname{tr}\left(A_{n-1, n-2} A_{n-2, n-1}\right), \\
& a_{n}=\operatorname{tr}\left(A_{n, n-2} A_{n-2, n}\right), \\
& X=\operatorname{tr}\left(A_{2,0} A_{1,2} A_{2,1} A_{0,2}\right), \\
& Y=\operatorname{tr}\left(A_{2,0} A_{3,2} \ldots A_{n-2, n-3} A_{n, n-2} A_{n-2, n} A_{n-3, n-2} \ldots A_{3,4} A_{2,3} A_{0,2}\right), \\
& Z=\operatorname{tr}\left(A_{2,0} A_{1,2} A_{2,1} A_{3,2} \ldots A_{n-2, n-3} A_{n, n-2} A_{n-2, n} A_{n-3, n-2} \ldots A_{3,4} A_{2,3} A_{0,2}\right) .
\end{aligned}
$$

In this Section 3.2 we will determine the minimal generators of $\mathbf{C}\left[\mu_{\mathbf{C}}^{-1}(Z)\right]^{G L(V, A, \alpha)}$ for Mckay quiver of type $\tilde{D}_{n}$. The graph of type $\tilde{D}_{n}$ has no loop. So the degrees of the traces of cycles are even. At first we consider the case of $n \geq 5$. Now we write down the condition that $\mu\left(\left\{A_{i, j}\right\}\right)$ is in $Z$ :

$$
\begin{aligned}
& A_{i+1, i} A_{i, i+1}-A_{i-1, i} A_{i, i-1} \\
& \quad=\frac{1}{2} \operatorname{tr}\left(A_{i+1, i} A_{i, i+1}-A_{i-1, i} A_{i, i-1}\right) E_{2} \quad(3 \leq i \leq n-3),
\end{aligned}
$$




$$
\begin{gathered}
A_{0,2} A_{2,0}+A_{1,2} A_{2,1}-A_{3,2} A_{2,3}=\frac{1}{2} \operatorname{tr}\left(A_{0,2} A_{2,0}+A_{1,2} A_{2,1}-A_{3,2} A_{2,3}\right) E_{2}, \\
A_{n, n-2} A_{n-2, n}+A_{n-1, n-2} A_{n-2, n-1}-A_{n-3, n-2} A_{n-2, n-3} \\
=\frac{1}{2} \operatorname{tr}\left(A_{n, n-2} A_{n-2, n}+A_{n-1, n-2} A_{n-2, n-1}-A_{n-3, n-2} A_{n-2, n-3}\right) E_{2} .
\end{gathered}
$$

From now on we will determine generators up to equivalence relation inductively on weight. From now on we use the word "weight" as the degree of homogeneous polynomial in polynomial $\operatorname{ring} \mathbf{C}[\mathfrak{R}(\mathbf{V}, \mathbf{A}, \alpha)]$. Any trace of cycle whose weight is 2 is $\operatorname{tr}\left(A_{i, j} A_{j, i}\right)$ for some edge $a=(i \bullet \rightarrow \bullet j)$. We put,

$$
A_{0}=\left\{A_{0,2}, A_{1,2}, A_{n-1, n-2}, A_{n, n-2}\right\} .
$$

Let $C_{4}$ be the set of cycles which contain neither $A_{i, i+1} A_{i+1, i}$ nor $A_{i+1, i} A_{i, i+1}(2 \leq i \leq n-3)$ and whose traces have weight $\geq 4$.

Lemma 1. The trace of cycle which has weight $\geq 4$ is equivalent to a sum of traces of elements of $C_{4}$.

Proof. The trace of cycle which has weight $2 l$ and contains no element of $A_{0}$ is equivalent to $\operatorname{tr}\left\{\left(A_{3,2} A_{2,3}\right)^{l}\right\}$ by relation (1). $\operatorname{tr}\left\{\left(A_{3,2} A_{2,3}\right)^{l}\right\}$ is equivalent to a union of traces of cycles which contain at least either $A_{0,2}$ or $A_{1,2}$ by (2). So it is necessary only to consider cycles which contain elements of $A_{0}$. In particular we have only to prove proposition in the case of cycles which start from an element of $A_{0}$. We order elements of $A_{0}$ in the cycle by the order of appearance in cycle. For example, for $A_{2,0} A_{3,2} \ldots A_{n-2, n-3} A_{n, n-2} A_{n-2, n} A_{n-3, n-2} \ldots A_{3,4} A_{2,3} A_{0.2}$, we put down $\alpha_{4} A_{3,2} \ldots A_{n-2, n-3} \alpha_{3} \alpha_{2} A_{n-3, n-2} \ldots A_{2,3} \alpha_{1}$. If the cycle contains $A_{i, i+1} A_{i+1, i}$ or $A_{i+1, i} A_{i, i+1} \quad(2 \leq i \leq n-3)$ between $\alpha_{i}$ and $\alpha_{i+1}$, by inductive use of (1). (2) the trace of the cycle is equivalent to a union of the traces of the cycles which contain neither $A_{i, i+1} A_{i+1, i}$ nor $A_{i+1, i} A_{i, i+1}(2 \leq i \leq n-3)$ between $\alpha_{i}$ and $\alpha_{i+1}$. Then lemma has been proved.

Lemma 2. If an element of $A_{0}$ appears two times in a cycle, the trace of it is equivalent to 0 .

Proof. It is obvious from the fact that $\alpha_{0}=\alpha_{1}=\alpha_{n-1}=\alpha_{n}=1$.

Lemma 3. If any element of $A_{0}$ appears at least one time in a cycle, then trace of it is equivalent to 0 .

Proof. By above lemmas up to equivalence the trace of cycle whose weight is bigger than 4 and not equal to $2(n-2), 2(n-1)$, nor $2 n$ is equivalent to 0 . Then it is enough to consider the following cycles:

$$
\begin{aligned}
& A_{2,0} A_{1,2} A_{2,1} A_{3,2} \ldots A_{n-2, n-3} A_{n-1, n-2} A_{n-2, n-1} A_{n, n-2} A_{n-2, n} A_{n-3, n-2} \ldots A_{3,4} A_{2,3} A_{0,2}, \\
& A_{2,0} A_{1,2} A_{2,1} A_{3,2} \ldots A_{n-2, n-3} A_{n, n-2} A_{n-2, n} A_{n-1, n-2} A_{n-2, n-1} A_{n-3, n-2} \ldots A_{3.4} A_{2,3} A_{0,2},
\end{aligned}
$$




$$
\begin{aligned}
& A_{2,0} A_{3,2} \ldots A_{n-2, n-3} A_{n-1, n-2} A_{n-2, n-1} A_{n, n-2} A_{n-2, n} A_{n-3, n-2} \ldots A_{3,4} A_{2,3} A_{1,2} A_{2,1} A_{0,2}, \\
& A_{2,0} A_{3,2} \ldots A_{n-2, n-3} A_{n, n-2} A_{n-2, n} A_{n-1, n-2} A_{n-2, n-1} A_{n-3, n-2} \ldots A_{3,4} A_{2,3} A_{1,2} A_{2,1} A_{0.2} .
\end{aligned}
$$

But the traces of these cycles are equivalent to 0 by inductive use of (1), (2), (3).

Proposition 3. The trace of cycle whose weight is bigger than 4 and not equal to $2(n-2)$ nor $2(n-1)$ is equivalent to 0 .

Proof. It is the summation of above lemmas.

Proposition 4. The trace of cycle whose weight is 4 is equivalent to

$$
X=\operatorname{tr}\left(A_{2,0} A_{1,2} A_{2,1} A_{0,2}\right)
$$

up to constant. The trace of cycle whose weight is $2(n-2)$ is equivalent to

$$
Y=\operatorname{tr}\left(A_{2,0} A_{3,2} \ldots A_{n-2, n-3} A_{n, n-2} A_{n-2, n} A_{n-3, n-2} \ldots A_{3,4} A_{2,3} A_{0,2}\right)
$$

up to constant. The trace of cycle whose weight is $2(n-1)$ is equivalent to

$$
Z=\operatorname{tr}\left(A_{2,0} A_{1,2} A_{2,1} A_{3,2} \ldots A_{n-2, n-3} A_{n, n-2} A_{n-2, n} A_{n-3, n-2} \ldots A_{3,4} A_{2,3} A_{0,2}\right)
$$

up to constant.

Proof. By above lemmas and easy calculation. At first we consider only cycles which begin from $A_{0,2}$. Then we have only two cycles whose traces have weight $2(n-2)$ and whose traces are not equivalent to 0 . i.e.

$$
\begin{gathered}
A_{2,0} A_{3,2} \ldots A_{n-2, n-3} A_{n, n-2} A_{n-2, n} A_{n-3, n-2} \ldots A_{3,4} A_{2,3} A_{0,2}, \\
A_{2,0} A_{3,2} \ldots A_{n-2, n-3} A_{n-1, n-2} A_{n-2, n-1} A_{n-3, n-2} \ldots A_{3,4} A_{2,3} A_{0,2} .
\end{gathered}
$$

But the traces of these cycles are equivalent by (3). We have only six cycles which begin from $A_{0,2}$ and whose traces have weight $2(n-1)$ and whose traces are not equivalent to 0 . i.e.

$$
\begin{gathered}
A_{2,0} A_{1,2} A_{2,1} A_{3,2} \ldots A_{n-2, n-3} A_{n, n-2} A_{n-2, n} A_{n-3, n-2} \ldots A_{3,4} A_{2,3} A_{0,2}, \\
A_{2,0} A_{3,2} \ldots A_{n-2, n-3} A_{n, n-2} A_{n-2, n} A_{n-3, n-2} \ldots A_{3,4} A_{2,3} A_{2,1} A_{1,2} A_{0,2}, \\
A_{2,0} A_{1,2} A_{2,1} A_{3,2} \ldots A_{n-2, n-3} A_{n-1, n-2} A_{n-2, n-1} A_{n-3, n-2} \ldots A_{3,4} A_{2,3} A_{0,2}, \\
A_{2,0} A_{3,2} \ldots A_{n-2, n-3} A_{n-1, n-2} A_{n-2, n-1} A_{n-3, n-2} \ldots A_{3,4} A_{2,3} A_{1,2} A_{2,1} A_{0,2}, \\
A_{2,0} A_{3,2} \ldots A_{n-2, n-3} A_{n-1, n-2} A_{n-2, n-1} A_{n, n-2} A_{n-2, n} A_{n-3, n-2} \ldots A_{3,4} A_{2,3} A_{0,2}, \\
A_{2,0} A_{3,2} \ldots A_{n-2, n-3} A_{n, n-2} A_{n-2, n} A_{n-1, n-2} A_{n-2, n-1} A_{n-3, n-2} \ldots A_{3,4} A_{2,3} A_{0,2} .
\end{gathered}
$$

The traces of these cycles are equivalent by (1), (2), (3). When we consider cycles which start from other 3 -elements of $A_{0}$ and whose traces have weight $\leq 4$, we can check by (1), (2), (3) that the traces of these cycles are equivalent to trace of above cycle. Then we obtain proposition. 
Next we consider the case of $n=4$. This case is slightly different from other cases, but more easy. So we write down only results. In this case the condition of $\mu\left(\left\{A_{i, j}\right\}\right) \in Z$ is as follows,

$$
\begin{aligned}
A_{0,2} A_{2,0}+A_{1,2} A_{2,1}-A_{3,2} A_{2,3}-A_{4,2} A_{2,4} \\
\quad=\frac{1}{2} \operatorname{tr}\left(A_{0,2} A_{2,0}+A_{1,2} A_{2,1}-A_{3,2} A_{2,3}-A_{4,2} A_{2,4}\right) E_{2} .
\end{aligned}
$$

Proposition 5. In the case of $n=4$ the generators of $\mathbf{C}\left[\mu_{\mathbf{C}}^{-1}(Z)\right]^{G L(V, A, x)}$ are just same elements as in the case of $n \geq 5$ up to equivalence, i.e.

$$
\begin{gathered}
a_{1}=\operatorname{tr}\left(A_{2,0} A_{0,2}\right), \\
a_{2}=\operatorname{tr}\left(A_{2,1} A_{1,2}\right), \\
a_{3}=\operatorname{tr}\left(A_{3,2} A_{2,3}\right), \\
a_{4}=\operatorname{tr}\left(A_{4,2} A_{2,4}\right), \\
X=\operatorname{tr}\left(A_{2,0} A_{1,2} A_{2,1} A_{0,2}\right), \\
Y=\operatorname{tr}\left(A_{2,0} A_{3,2} A_{2,3} A_{0,2}\right), \\
Z=\operatorname{tr}\left(A_{2,0} A_{1,2} A_{2,1} A_{3,2} A_{2,3} A_{0,2}\right) .
\end{gathered}
$$

Next we state how to find a relation between these generators $(n \geq 4)$. At first we introduce following notation,

$$
\begin{gathered}
A:=A_{0,2} A_{2,0}, \\
B:=A_{1,2} A_{2,1}, \\
C:=A_{3,2} \ldots A_{n-2, n-3} A_{n, n-2} A_{n-2, n} A_{n-3, n-2} \ldots A_{2,3} .
\end{gathered}
$$

Since these elements are $2 \times 2$-matrices, the relation of Theorem 3 gives the relation between generators $\left\{a_{1}, \ldots, a_{n}, X, Y, Z\right\}$ of $\mathbf{C}\left[\mu_{\mathbf{C}}^{-1}(Z)\right]^{G L(V, A, x)}$. If this relation is irreducible, it is the unique relation because of $\operatorname{dim} \mu_{\mathrm{C}}^{-1}(Z) / / G L(V, A, \alpha) \geq(n+2)$. It will be proved in appendix that this relation is irreducible.

\section{3. type $\tilde{E}_{6}$}

Theorem 6. For Mckay quiver of type $\tilde{E}_{6} \mathbf{C}\left[\mu_{\mathbf{C}}^{-1}(Z)\right]^{G L(1: A . x)}$ is generated by $(n+3)$ generators and there is a unique irreducible relation between these generators. Moreover this relation is a quasihomogeneous polynomial with weight $(24 ; 2, \ldots, 2,6$, 8, 12). In particular $\mathbf{C}\left[\mu_{\mathbf{C}}^{-1}(0)\right]^{G L(V, A, \alpha)}$ is generated by 3 generators and the relation between these generators represents simple singularity of type $E_{6}$. We write down generators.

$$
\begin{aligned}
& a_{1}=\operatorname{tr}\left(A_{1,0} A_{0,1}\right), \\
& a_{2}=\operatorname{tr}\left(A_{2,1} A_{1,2}\right), \\
& a_{3}=\operatorname{tr}\left(A_{3,2} A_{2,3}\right),
\end{aligned}
$$




$$
\begin{gathered}
a_{4}=\operatorname{tr}\left(A_{4,3} A_{3,4}\right), \\
a_{5}=\operatorname{tr}\left(A_{5,2} A_{2,5}\right), \\
a_{6}=\operatorname{tr}\left(A_{6,5} A_{5,6}\right), \\
X:=\operatorname{tr}\left(A_{3,2} A_{2,3} A_{5,2} A_{2,5} A_{5,2} A_{2,5}\right), \\
Y:=\operatorname{tr}\left(A_{3,2} A_{2,3} A_{5,2} A_{2,5} A_{3,2} A_{2,3} A_{5,2} A_{2,5}\right), \\
Z:=\operatorname{tr}\left(A_{3,2} A_{2,3} A_{5,2} A_{2,5} A_{3,2} A_{2,3} A_{3,2} A_{2,3} A_{5,2} A_{2,5} A_{5,2} A_{2,5}\right) .
\end{gathered}
$$

The graph of type $\tilde{E}_{6}$ has no loop. So the degrees of traces of cycles are even. The generators with weight 2 correspond to each arrow similarly as in the case of type $\tilde{D}_{n}$. Now we write down the condition that $\mu\left(\left\{A_{i, j}\right\}\right)$ is in $Z$ :

$$
\begin{gathered}
A_{i+1, i} A_{i, i+1}-A_{i-1, i} A_{i, i-1}=\frac{1}{2} \operatorname{tr}\left(A_{i+1, i} A_{i, i+1}-A_{i-1, i} A_{i, i-1}\right) E_{2} \quad(i=1,3,5) \\
A_{3,2} A_{2,3}+A_{2,5} A_{5,2}-A_{1,2} A_{2,1}=\frac{1}{3} \operatorname{tr}\left(A_{3,2} A_{2,3}+A_{2,5} A_{5,2}-A_{1,2} A_{2,1}\right) E_{3}
\end{gathered}
$$

We put,

$$
\begin{aligned}
A & :=A_{3,2} A_{2,3}, \\
B & :=A_{5,2} A_{2,5}, \\
C & :=A_{1.2} A_{2,1} .
\end{aligned}
$$

From these relations next lemma follows:

Lemma 4. The generators with weight $\geq 4$ are equivalent to following traces up to equivalence:

$$
\operatorname{tr}\left(A^{i_{1}} B^{j_{1}} \ldots A^{i_{k}} B^{j_{k}}\right) \quad\left(k>0,1 \leq i_{s}, j_{s} \leq 2\right) .
$$

We have following equivalences for $l \geq 2$ :

$$
\operatorname{tr}\left(A^{l}\right) \sim \operatorname{tr}\left(B^{l}\right) \sim \operatorname{tr}\left(C^{l}\right) \sim \operatorname{tr}\left\{(A+B)^{l}\right\} \sim 0 .
$$

In particular:

$$
\begin{gathered}
\operatorname{tr}\left\{(A+B)^{2}\right\} \sim 2 \operatorname{tr}(A B) \sim 0, \\
\operatorname{tr}\left\{(A+B)^{3}\right\} \sim 3\left\{\operatorname{tr}\left(A^{2} B\right)+\operatorname{tr}\left(A B^{2}\right)\right\} \sim 0 .
\end{gathered}
$$

By these equivalences:

$$
\operatorname{tr}(A B) \sim 0, \quad \operatorname{tr}\left(A^{2} B\right) \sim-\operatorname{tr}\left(A B^{2}\right)
$$

Since $A, B, C$ are $3 \times 3$-matrices, by Theorem 4 we have only to consider followings as generators with weight $\geq 4$ of $\mathbf{C}\left[\mu_{\mathbf{C}}^{-1}(Z)\right]^{G L(V, A, \alpha)}$,

$$
\left\{\operatorname{det} A, \operatorname{det} B, \operatorname{tr}(A B), \operatorname{tr}\left(A^{2}\right), \operatorname{tr}\left(B^{2}\right), \operatorname{tr}\left(A^{2} B\right), \operatorname{tr}\left(A B^{2}\right), \operatorname{tr}(A B A B), \operatorname{tr}\left(A B A^{2} B^{2}\right)\right\} .
$$


Hence by above calculation we have only to consider

$$
\left\{X:=\operatorname{tr}\left(A B^{2}\right), Y:=\operatorname{tr}(A B A B), Z:=\operatorname{tr}\left(A B A^{2} B^{2}\right)\right\}
$$

as generators with weight $\geq 4$ of $\mathbf{C}\left[\mu_{\mathbf{C}}^{-1}(Z)\right]^{G L(V, A, x)}$. Since these elements are $(3,3)$-matrices, the relation of Theorem 4 gives the relation between generators $\left\{a_{1}, \ldots, a_{6}, X, Y, Z\right\}$ of $\mathbf{C}\left[\mu_{\mathbf{C}}^{-1}(Z)\right]^{G L(V, A, x)}$. If this relation is irreducible, it is the unique relation because of $\operatorname{dim} \mu_{\mathbf{C}}^{-1}(Z) / / G L(V, A, \alpha) \geq(n+2)$. It will be proved in appendix that this relation is irreducible.

Remark 2. In this case we used vertex '2' as start point of cycles. So we used the invariant theory of $(3,3)$-matrices. When we use vertex ' 1 ' as start point of cycles, we can use the invariant theory of $(2,2)$-matrices.

\section{4. type $\tilde{E}_{7}$}

Theorem 7. For Mckay quiver of type $\tilde{E}_{7} \mathbf{C}\left[\mu_{\mathbf{C}}^{-1}(Z)\right]^{G L(V, A, \alpha)}$ is generated by $(n+3)$ generators and there is a unique irreducible relation between these generators. Moreover this relation is a quasihomogeneous polynomial with weight $(36 ; 2, \ldots, 2$, $8,12,18)$. In particular $\mathbf{C}\left[\mu_{\mathbf{C}}^{-1}(0)\right]^{G L(V, A, \alpha)}$ is generated by 3 generators and the relation between these generators represents simple singularity of type $E_{7}$. We write down generators.

$$
\begin{aligned}
& a_{1}=\operatorname{tr}\left(A_{1,0} A_{0,1}\right), \\
& a_{2}=\operatorname{tr}\left(A_{2,1} A_{1,2}\right), \\
& a_{3}=\operatorname{tr}\left(A_{3,2} A_{2,3}\right), \\
& a_{4}=\operatorname{tr}\left(A_{4,3} A_{3,4}\right), \\
& a_{5}=\operatorname{tr}\left(A_{5,4} A_{4,5}\right), \\
& a_{6}=\operatorname{tr}\left(A_{6,5} A_{5,6}\right), \\
& a_{7}=\operatorname{tr}\left(A_{7,3} A_{3,7}\right),
\end{aligned}
$$

$X:=\operatorname{tr}\left(A_{3,7} A_{4,3} A_{5,4} A_{6,5} A_{5,6} A_{4,5} A_{3,4} A_{7,3}\right)$,

$Y:=\operatorname{tr}\left(A_{3,7} A_{4,3} A_{5,4} A_{6,5} A_{5,6} A_{4,5} A_{3,4} A_{7,3} A_{3,7} A_{4,3} A_{3,4} A_{7,3}\right)$,

$Z:=\operatorname{tr}\left(A_{3,7} A_{4,3} A_{3,4} A_{7,3} A_{3,7} A_{4,3} A_{5,4} A_{4,5} A_{3,4} A_{7,3} A_{3,7} A_{4,3} A_{5,4} A_{6,5} A_{5,6} A_{4,5} A_{3,4} A_{7,3}\right)$.

The graph of Type $\tilde{E}_{7}$ has no loop. So the degrees of traces of cycles are even. The generators with weight 2 are corresponding to each arrow similarly as in the case of type $\tilde{D}_{n}$. Now we write down the condition that $\mu\left(\left\{A_{i, j}\right\}\right)$ is in $Z$ :

$$
\begin{array}{ll}
A_{i+1, i} A_{i, i+1}-A_{i-1, i} A_{i, i-1}=\frac{1}{2} \operatorname{tr}\left(A_{i+1, i} A_{i, i+1}-A_{i-1, i} A_{i, i-1}\right) E_{2} & (i=1,5), \\
A_{i+1, i} A_{i, i+1}-A_{i-1, i} A_{i, i-1}=\frac{1}{3} \operatorname{tr}\left(A_{i+1, i} A_{i, i+1}-A_{i-1, i} A_{i, i-1}\right) E_{3} & (i=2,4),
\end{array}
$$




$$
\begin{gathered}
A_{3,7} A_{7,3}=\frac{1}{2} \operatorname{tr}\left(A_{3,7} A_{7,3}\right) E_{2} \\
A_{7,3} A_{3,7}+A_{4,3} A_{3,4}-A_{2,3} A_{3,2}=\frac{1}{4} \operatorname{tr}\left(A_{7,3} A_{3,7}+A_{4,3} A_{3,4}-A_{2,3} A_{3,2}\right) E_{4}
\end{gathered}
$$

We put,

$$
\begin{aligned}
A & :=A_{7,3} A_{3,7}, \\
B & :=A_{4,3} A_{3,4}, \\
C & :=A_{2,3} A_{3,2} .
\end{aligned}
$$

From these relations next lemma follows.

Lemma 5. The generators with weight $\geq 4$ are as follows up to equivalence,

$$
\operatorname{tr}\left(A B^{i_{1}} \ldots A B^{i_{k}}\right) \quad\left(1 \leq i_{s} \leq 3\right) .
$$

We put,

$$
\begin{gathered}
\alpha:=A_{3,7} A_{4,3} A_{3,4} A_{7,3}, \\
\beta:=A_{3,7} A_{4,3} A_{5,4} A_{4,5} A_{3,4} A_{7,3}, \\
\gamma:=A_{3,7} A_{4,3} A_{5,4} A_{6,5} A_{5,6} A_{4,5} A_{3,4} A_{7,3} .
\end{gathered}
$$

Then $\alpha, \beta, \gamma$ are $2 \times 2$-matrices. Hence above lemma is restated as follows.

Lemma 6. The generators with weight $\geq 4$ are the traces of monomials of $\alpha, \beta, \gamma$ up to equivalence.

As in the case of $\tilde{E}_{6}$, for $l \geq 2$ we have:

$$
\operatorname{tr}\left(A^{l}\right) \sim \operatorname{tr}\left(B^{\prime}\right) \sim \operatorname{tr}\left\{(A+B)^{\prime}\right\} \sim 0 .
$$

In particular

$$
\begin{gathered}
\operatorname{tr}\left\{(A+B)^{2}\right\} \sim 2 \operatorname{tr}(A B) \sim 0, \\
\operatorname{tr}\left\{(A+B)^{3}\right\} \sim 3 \operatorname{tr}\left(A B^{2}\right) \sim 0, \\
\operatorname{tr}\left\{(A+B)^{4}\right\} \sim 4 \operatorname{tr}\left(A B^{3}\right)+2 \operatorname{tr}(A B A B) \sim 0, \\
\operatorname{tr}\left\{(A+B)^{5}\right\} \sim 5 \operatorname{tr}\left(A B A B^{2}\right) \sim 0, \\
\operatorname{tr}\left\{(A+B)^{6}\right\} \sim 3 \operatorname{tr}\left(A B^{2} A B^{2}\right)+6 \operatorname{tr}\left(A B A B^{3}\right)+2 \operatorname{tr}(A B A B A B) \sim 0, \\
\operatorname{tr}\left\{(A+B)^{7}\right\} \sim 7 \operatorname{tr}\left(A B^{2} A B^{3}\right)+7 \operatorname{tr}\left(A B A B A B^{2}\right) \sim 0 .
\end{gathered}
$$

Then moreover we have following equivalence relations:

$$
\begin{gathered}
\operatorname{tr} \alpha \sim \operatorname{tr}(A B) \sim 0, \\
\operatorname{tr} \beta \sim \operatorname{tr}\left(A B^{2}\right) \sim 0, \\
2 \operatorname{tr} \gamma+\operatorname{tr}\left(\alpha^{2}\right) \sim 2 \operatorname{tr}\left(A B^{3}\right)+\operatorname{tr}(A B A B) \sim 0,
\end{gathered}
$$




$$
\begin{gathered}
\operatorname{tr}(\alpha \beta) \sim \operatorname{tr}\left(A B A B^{2}\right) \sim 0, \\
3 \operatorname{tr}\left(\beta^{2}\right)+6 \operatorname{tr}(\alpha \gamma)+2 \operatorname{tr}\left(\alpha^{3}\right) \sim 3 \operatorname{tr}\left(A B^{2} A B^{2}\right)+6 \operatorname{tr}\left(A B A B^{3}\right)+2 \operatorname{tr}(A B A B A B) \sim 0, \\
\operatorname{tr}(\beta \gamma)+\operatorname{tr}\left(\alpha^{2} \beta\right) \sim \operatorname{tr}\left(A B^{2} A B^{3}\right)+\operatorname{tr}\left(A B A B A B^{2}\right) \sim 0 .
\end{gathered}
$$

Hence we have by Cayley-Hamilton equation: $\alpha^{2}-(\operatorname{tr} \alpha) \alpha+(\operatorname{det} \alpha) E_{2}=0$,

$$
\begin{aligned}
\operatorname{tr}\left(\alpha^{3}\right) & =\operatorname{tr}\left[\alpha\left\{(\operatorname{tr} \alpha) \alpha-(\operatorname{det} \alpha) E_{2}\right\}\right] \\
& =\operatorname{tr}\left(\alpha^{2}\right) \operatorname{tr} \alpha-(\operatorname{det} \alpha) \operatorname{tr} \alpha \sim 0, \\
\operatorname{tr}\left(\alpha^{2} \beta\right) & =\operatorname{tr}\left[\beta\left\{(\operatorname{tr} \alpha) \alpha-(\operatorname{det} \alpha) E_{2}\right\}\right] \\
& =\operatorname{tr}(\alpha \beta) \operatorname{tr} \alpha-(\operatorname{det} \alpha) \operatorname{tr} \beta \sim 0 .
\end{aligned}
$$

When we combine above relations,

$$
\begin{gathered}
\operatorname{tr}\left(\beta^{2}\right)+2 \operatorname{tr}(\alpha \gamma) \sim 0, \\
-\operatorname{tr}\left(\alpha^{2} \beta\right) \sim \operatorname{tr}(\beta \gamma) \sim 0 .
\end{gathered}
$$

Since $\{\alpha, \beta, \gamma\}$ are $2 \times 2$-matrices, by Theorem 3 the generators with weight $\geq 4$ are followings up to equivalence,

$$
\begin{gathered}
X:=\operatorname{tr}(\gamma), \\
Y:=\operatorname{tr}(\gamma \alpha), \\
Z:=\operatorname{tr}(\alpha \beta \gamma) .
\end{gathered}
$$

Because these elements are $2 \times 2$-matrices, the relation of Theorem 3 gives the relation between generators $\left\{a_{1}, \ldots, a_{7}, X, Y, Z\right\}$ of $\mathbf{C}\left[\mu_{\mathbf{C}}^{-1}(Z)\right]^{G L(V, A, \alpha)}$. If this relation is irreducible, it is the unique relation because of $\operatorname{dim}_{\mathbf{C}}^{-1}(Z) / /$ $G L(V, A, \alpha) \geq(n+2)$. It will be proved in appendix that this relation is irreducible.

\section{5. type $\tilde{E}_{8}$}

Theorem 8. For Mckay quiver of type $\tilde{E}_{8} \mathbf{C}\left[\mu_{\mathbf{C}}^{-1}(Z)\right]^{G L(V, A, \alpha)}$ is generated by $(n+3)$ generators and have a unique irreducible relation between these generators. Moreover this relation is a quasihomogeneous polynomial with weight $(60 ; 2, \ldots, 2$. 12, 20,30). In particular $\mathbf{C}\left[\mu_{\mathbf{C}}^{-1}(0)\right]^{G L(V, A, \alpha)}$ is generated by 3 generators and the relation between these generators represents simple singularity of type $E_{8}$. We write down generators.

$$
\begin{aligned}
& a_{1}=\operatorname{tr}\left(A_{1,0} A_{0,1}\right), \\
& a_{2}=\operatorname{tr}\left(A_{2,1} A_{1,2}\right), \\
& a_{3}=\operatorname{tr}\left(A_{3,2} A_{2,3}\right), \\
& a_{4}=\operatorname{tr}\left(A_{4,3} A_{3,4}\right),
\end{aligned}
$$




$$
\begin{gathered}
a_{5}=\operatorname{tr}\left(A_{5,4} A_{4,5}\right) \\
a_{6}=\operatorname{tr}\left(A_{6,5} A_{5,6}\right) \\
a_{7}=\operatorname{tr}\left(A_{7,6} A_{6,7}\right) \\
a_{8}=\operatorname{tr}\left(A_{8,5} A_{5,8}\right) \\
X:=\operatorname{tr}\left(A_{5,8} A_{6,5} A_{7,6} A_{6,7} A_{5,6} A_{8,5} A_{5,8} A_{6,5} A_{7,6} A_{6,7} A_{5,6} A_{8,5}\right), \\
Y:=\operatorname{tr}\left(A_{5,8} A_{6,5} A_{5,6} A_{8,5} A_{5,8} A_{6,5} A_{7,6} A_{6,7} A_{5,6} A_{8,5} A_{5,8} A_{6,5} A_{5,6} A_{8,5}\right. \\
\left.A_{5,8} A_{6,5} A_{7,6} A_{6,7} A_{5,6} A_{8,5}\right) \\
Z:=\operatorname{tr}\left(A_{5,8} A_{6,5} A_{5,6} A_{8,5} A_{5,8} A_{6,5} A_{7,6} A_{6,7} A_{5,6} A_{8,5} A_{5,8} A_{6,5} A_{5,6} A_{8,5}\right. \\
\left.A_{5,8} A_{6,5} A_{5,6} A_{8,5} A_{5,8} A_{6,5} A_{7,6} A_{6,7} A_{5,6} A_{8,5} A_{5,8} A_{6,5} A_{7,6} A_{6,7} A_{5,6} A_{8,5}\right) .
\end{gathered}
$$

The graph of type $\tilde{E}_{8}$ has no loop. So the degrees of traces of cycles are even. The generators with weight 2 are corresponding to each arrow similarly as other cases. Now we write down the condition of $\mu\left(\left\{A_{i, j}\right\}\right) \in Z$,

$$
\begin{gathered}
A_{i+1, i} A_{i, i+1}-A_{i-1, i} A_{i, i-1}=\frac{1}{3} \operatorname{tr}\left(A_{i+1, i} A_{i, i+1}-A_{i-1, i} A_{i, i-1}\right) E_{k_{i}}, \\
\left(i=1,2,3,4,6 \text { and } k_{i}=2,3,4,5,4 \text { for each } i\right), \\
A_{6,7} A_{7,6}=\frac{1}{2} \operatorname{tr}\left(A_{6,7} A_{7,6}\right) E_{2} \\
A_{5,8} A_{8,5}=\frac{1}{3} \operatorname{tr}\left(A_{5,8} A_{8,5}\right) E_{3} \\
A_{6,5} A_{5,6}+A_{8,5} A_{5,8}-A_{4,5} A_{5,4}=\frac{1}{6} \operatorname{tr}\left(A_{6,5} A_{5,6}+A_{8,5} A_{5,8}-A_{4,5} A_{5,4}\right) E_{6} .
\end{gathered}
$$

We put,

$$
\begin{aligned}
A & :=A_{8,5} A_{5,8}, \\
B & :=A_{6,5} A_{5,6}, \\
C & :=A_{4,5} A_{5,4} .
\end{aligned}
$$

From these relations next lemma follows.

Lemma 7. The generators with weight $\geq 4$ are equivalent to following traces,

$$
\operatorname{tr}\left(A B^{i_{1}} \ldots A B^{i_{k}}\right) \quad\left(1 \leq i_{s} \leq 2\right) .
$$

We put,

$$
\begin{gathered}
\alpha:=A_{5,8} A_{6,5} A_{5,6} A_{8,5} . \\
\beta:=A_{5,8} A_{6,5} A_{7,6} A_{6,7} A_{5,6} A_{8,5} .
\end{gathered}
$$


$\{\alpha, \beta\}$ are $3 \times 3$-matrices. Similarly as in other cases the generators with weight $\geq 4$ are equivalent to,

$$
\begin{gathered}
X:=\operatorname{tr}\left(\beta^{2}\right), \\
Y:=\operatorname{tr}(\alpha \beta \alpha \beta), \\
Z:=\operatorname{tr}\left(\alpha \beta \alpha^{2} \beta^{2}\right) .
\end{gathered}
$$

Since these elements are $3 \times 3$-matrices, the relation of Theorem 4 gives the relation between generators $\left\{a_{1}, \ldots, a_{8}, X, Y, Z\right\}$ of $\mathbf{C}\left[\mu_{\mathbf{C}}^{-1}(Z)\right]^{G L(V, A, x)}$. If this relation is irreducible, it is the unique relation because of $\operatorname{dim} \mu_{\mathrm{C}}^{-1}(Z) / / G L(V, A, \alpha) \geq(n+2)$. It will be proved in appendix that this relation is irreducible.

\section{Appendix}

In this appendix we will prove that the relation between generators of $\mathbf{C}\left[\mu_{\mathbf{C}}^{-1}(Z)\right]^{G L(V, A, x)}$ is irreducible. The method of the proof are similar in the all cases. So we give the detail of the proof only in the case of type $\tilde{D}_{n}$. In the other cases we give only the outline. It is not nessesary for proving irreducibility to denote equation explicitly. But it is possible. So as a example we will write down the equation explicitly in the case of type $\tilde{D}_{n}$.

4.1. type $\tilde{D}_{n}$. At first we consider the case $n \geq 5$. Since we want to use the relation of Theorem 3 in order to determine the relation between the generators $\left\{a_{1}, \ldots, a_{n}, X, Y, Z\right\}$, we write the following generators of Theorem 3,

$\{\operatorname{det} A, \operatorname{det} B, \operatorname{det} C, \operatorname{tr} A, \operatorname{tr} B, \operatorname{tr} C, \operatorname{tr}(A B), \operatorname{tr}(B C), \operatorname{tr}(C A), \operatorname{tr}(A B C)\}$

by the generators $\left\{a_{1}, \ldots, a_{n}, X, Y, Z\right\}$ of $\mathbf{C}\left[\mu_{\mathbf{C}}^{-1}(Z)\right]^{G L(V \cdot A, x)}$ in the $D_{n}$-case $(n \geq 5)$.

By definition of $A, B, C$ we have:

$$
\begin{gathered}
\operatorname{det} A=\operatorname{det} B=\operatorname{det} C=0, \\
\operatorname{tr}(A B)=X, \\
\operatorname{tr}(A C)=Y, \\
\operatorname{tr}(A B C)=Z .
\end{gathered}
$$

So we have only to describe $\operatorname{tr}(C)$ and $\operatorname{tr}(B C)$ by $X, Y, Z, a_{1}, \ldots, a_{n}$. We put,

$$
\begin{gathered}
\Gamma_{k}:=\operatorname{tr}\left\{A_{n, n-2} A_{n-2, n}\left(A_{n-3, n-2} A_{n-2, n-3}\right)^{k}\right\}, \\
f_{k}:=\sum_{3 \leq l_{1}<\cdots<l_{k-1} \leq n-3} \frac{\left(-\sum_{i=3}^{n-3} \lambda_{i}\right) \cdots\left(-\sum_{i=n-3}^{n-3} \lambda_{i}\right)}{\left(-\sum_{i=l_{1}}^{n-3} \lambda_{i}\right) \cdots\left(-\sum_{i=l_{k-1}}^{n-3} \lambda_{i}\right)} \\
(k=1, \ldots, n-4)
\end{gathered}
$$


where

$$
\lambda_{i}:=\frac{1}{2}\left(a_{i+1}-a_{i}\right) \quad(i=3, \ldots, n-3) .
$$

Hence

$$
\operatorname{tr}(C)=\sum_{k=1}^{n-4} f_{k} \Gamma_{k}
$$

We write down $\Gamma_{k}$.

$$
\Gamma_{k}=\sum_{l=0}^{k}\left(-\lambda_{n-2}\right)^{k-l}{ }_{l+1} H_{k-1} \sum_{\alpha=1}^{[l / 2+1]} \sum_{\left\{0 \leq s_{i}, t_{j}(i \neq 1), 0 \leq s_{1}, \sum_{i=1}^{\alpha}\left(s_{i}+t_{i}\right)-1=l\right\}} \varphi_{\alpha}^{s_{1}, \ldots, t_{\alpha}}
$$

where

$$
\begin{aligned}
& \lambda_{n-2}=\frac{1}{2}\left(a_{n}+a_{n-1}-a_{n-2}\right) . \\
& l+1 H_{k-l}=k ! / l !(k-l-1) ! .
\end{aligned}
$$

Suppose $s_{1} \neq 0$, then we have

$$
\varphi_{\alpha}^{s_{1}, \ldots, t_{\alpha}}=a_{n-1}^{\left(\sum_{i=1}^{x} s_{i}-k\right)} a_{n}^{\left(\sum_{i=1}^{x} t_{i}-k\right)}\left(\operatorname{tr}\left(A_{n-1, n-2} A_{n-2, n-1} A_{n, n-2} A_{n-2, n}\right)^{k} .\right.
$$

In the case of $s_{1}=0$ we have

$$
\varphi_{\alpha}^{s_{1}, \ldots, t_{x}}=a_{n-1}^{\left(\sum_{i=1}^{x} s_{i}-k+1\right)} a_{n}^{\left(\sum_{i=1}^{x} t_{i}-k+1\right)}\left(\operatorname{tr}\left(A_{n-1, n-2} A_{n-2, n-1} A_{n, n-2} A_{n-2, n}\right)^{k-1} .\right.
$$

Next we must calculate $\operatorname{tr}\left(A_{n-1, n-2} A_{n-2, n-1} A_{n, n-2} A_{n-2, n}\right)$. At first:

$$
\operatorname{tr}\left(A_{2,3} A_{3,2} A_{2,3} A_{3,2}\right)=2 X+\frac{1}{2}\left(a_{1}^{2}+a_{2}^{2}+a_{3}^{2}\right)-a_{1} a_{2} .
$$

So:

$$
\begin{aligned}
\operatorname{tr}\left(A_{n-2, n-3} A_{n-3, n-2} A_{n-2, n-3} A_{n-3, n-2}\right) & =\frac{1}{2}\left(a_{n-2}^{2}-a_{3}^{2}\right)+\operatorname{tr}\left(A_{2,3} A_{3,2} A_{2,3} A_{3,2}\right) \\
& =2 X-a_{1} a_{2}+\frac{1}{2}\left(a_{1}^{2}+a_{2}^{2}+a_{n-2}^{2}\right) .
\end{aligned}
$$

Moreover:

$$
\operatorname{tr}\left(A_{n-1, n-2} A_{n-2, n-1} A_{n, n-2} A_{n-2, n}\right)=X+\frac{1}{4}\left(a_{1}^{2}+a_{2}^{2}-a_{n-1}^{2}+a_{n}^{2}\right)-\frac{1}{2}\left(a_{1} a_{2}-a_{n} a_{n-1}\right) .
$$

So we determined $\operatorname{tr}(C)$. Next we describe $\operatorname{tr}(B C)$. By similar calculation we have:

$$
\operatorname{tr}(B C)=\frac{1}{2}\left(a_{1}+a_{2}-a_{n-2}\right) \operatorname{tr}(C)-Y+\sum_{k=1}^{n-4} f_{k} \Gamma_{k+1}
$$


We determined $\operatorname{tr}(B C)$. Hence we determined a relation between generators $\left\{a_{1}, \ldots, a_{n}, X, Y, Z\right\}$ from the relation of theorem 3 . We write down this equation.

$$
\begin{aligned}
0= & Z^{2}-Z\left\{\frac{1}{2}\left(2 X+a_{1}^{2}-a_{1} a_{2}-a_{1} a_{n-2}\right) \sum_{k=1}^{n-4} f_{k} \Gamma_{k}-\left(a_{1}-a_{2}\right) Y+a_{1} \sum_{k=1}^{n-4} f_{k} \Gamma_{k+1}\right\} \\
& +X Y\left\{\frac{1}{2}\left(a_{1}+a_{2}-a_{n-2}\right) \sum_{k=1}^{n-4} f_{k} \Gamma_{k}-Y+\sum_{k=1}^{n-4} f_{k} \Gamma_{k+1}\right\} .
\end{aligned}
$$

When we look at this relation with respect to weight, we can find that this relation is given by a quasihomogeneous polynomial. In the case of type $D_{4}$ we can write down a relation of generators $\left\{a_{1}, \ldots, a_{4}, X, Y, Z\right\}$ by similar calculation:

$$
\begin{aligned}
0= & Z^{2}-Z\left\{X\left(a_{1}+a_{3}\right)+Y\left(a_{2}-a_{1}\right)-a_{1} a_{2} a_{3}+a_{1} a_{4}^{2}-\frac{1}{2} a_{1} a_{4}\left(a_{4}+a_{3}-a_{2}-a_{1}\right)\right. \\
& \left.+\frac{1}{4} a_{1}\left[\left(a_{1}-a_{2}\right)^{2}-\left(a_{3}-a_{4}\right)^{2}\right]\right\}+X^{2} Y-X Y^{2}+a_{4}^{2} X Y \\
& -\frac{1}{2} a_{4} X Y\left(a_{4}+a_{3}-a_{2}-a_{1}\right)+\frac{1}{4} X Y\left\{\left(a_{1}-a_{2}\right)^{2}-\left(a_{3}-a_{4}\right)^{2}\right\} .
\end{aligned}
$$

$\mathbf{C}\left[\mu_{\mathbf{C}}^{-1}(0)\right]^{G L(V, A, x)}$ is the ring which is quotient of $\mathbf{C}\left[\mu_{\mathbf{C}}^{-1}(Z)\right]^{G L(V, A, x)}$ by ideal $\left(a_{1}, \ldots, a_{n}\right)$. Thus $\mathbf{C}\left[\mu_{\mathbf{C}}^{-1}(0)\right]^{G L(V, A, x)}$ is generated by $X, Y, Z$. Hence by calculation: if $n$ is odd,

$$
\begin{gathered}
\operatorname{tr} C=(\operatorname{tr}(A B))^{(n-3) / 2}, \\
\operatorname{tr}(C A)=-\operatorname{tr}(B C),
\end{gathered}
$$

if $n$ is even,

$$
\begin{gathered}
\operatorname{tr} C=0, \\
\operatorname{tr}(C A)=-\operatorname{tr}(B C)+(\operatorname{tr}(A B))^{(n-2) / 2} .
\end{gathered}
$$

Moreover the unique relation between $X, Y, Z$ is:

$$
\begin{array}{cc}
Z^{2}-X^{(n-1) / 2} Z-X Y^{2}=0 & (n: \text { odd }), \\
Z^{2}+X^{n / 2}-X Y^{2}=0 & (n: \text { even }) .
\end{array}
$$

These relations are irreducible and represent simple singularity of type $D_{n}$. These equations are obtained from equation (16), (17) by restriction to

$$
\left\{a_{1}=\cdots=a_{n}=0\right\}
$$

Hence if equations (16), (17) are not irreducible, equations (18), (19) are not irreducible either. But it is contradiction. So equations (16), (17) are irreducible. 
4.2. type $\tilde{E}_{6}$. We want to use the relation of Theorem 4 in order to determine the relation between generators $\left\{a_{1}, \ldots, a_{n}, X, Y, Z\right\}$. Hence we have only to express generators of Theorem 4 by generators $\left\{a_{1}, \ldots, a_{6}, X, Y, Z\right\}$ of $\mathbf{C}\left[\mu_{\mathbf{C}}^{-1}(0)\right]^{G L(V, A, \alpha)}$. This case is more easy than the case of type $\tilde{D}_{n}$, because the method in determining generators in this case includes no inductive method. At first we only write down the result.

$$
\begin{gathered}
\operatorname{det} A=\operatorname{det} B=0, \\
\operatorname{tr} A=a_{3}, \\
\operatorname{tr} B=a_{5}, \\
\operatorname{tr} A B=\frac{1}{24}\left(3 a_{1}^{2}-a_{2}^{2}-2 a_{3}^{2}-6 a_{4}^{2}-2 a_{5}^{2}-6 a_{6}^{2}+8 a_{3} a_{5}\right), \\
\operatorname{tr}\left(A^{2}\right)=\frac{1}{2}\left(a_{3}^{2}+a_{4}^{2}\right), \\
\operatorname{tr}\left(B^{2}\right)=\frac{1}{2}\left(a_{5}^{2}+a_{6}^{2}\right), \\
+\frac{1}{8}\left(a_{2}^{2}+a_{1}\right)^{3}+\frac{1}{8}\left(a_{2}-a_{1}\right)^{3}-\frac{1}{8}\left(a_{3}+a_{4}\right)^{3}-\frac{1}{8}\left(a_{3}-a_{4}\right)^{3} \\
-\frac{1}{8}\left(a_{5}+a_{6}\right)^{3}-\frac{1}{8}\left(a_{5}-a_{6}\right)^{3}-3 X, \\
\operatorname{tr}\left(A B^{2}\right)=X, \\
\operatorname{tr}(A B A B)=Y, \\
\operatorname{tr}\left(A B A^{2} B^{2}\right)=Z .
\end{gathered}
$$

We will explain the method to find these equalities. At first by relation (8):

$$
\begin{aligned}
\operatorname{tr}\left(A^{n}\right) & =\operatorname{tr}\left\{A_{4,3} A_{3,4}+\frac{1}{2}\left(a_{3}-a_{4}\right) E_{2}\right\}^{n} \\
& =\frac{1}{2^{n}}\left(a_{3}+a_{4}\right)^{n}+\frac{1}{2^{n}}\left(a_{3}-a_{4}\right)^{n}, \\
\operatorname{tr}\left(B^{n}\right) & =\operatorname{tr}\left\{A_{6,5} A_{5,6}+\frac{1}{2}\left(a_{5}-a_{6}\right) E_{2}\right\}^{n} \\
& =\frac{1}{2^{n}}\left(a_{5}+a_{6}\right)^{n}+\frac{1}{2^{n}}\left(a_{5}-a_{6}\right)^{n} . \\
\operatorname{tr}\left(C^{n}\right) & =\operatorname{tr}\left\{A_{0,1} A_{1,0}+\frac{1}{2}\left(a_{2}-a_{1}\right) E_{2}\right\}^{n} \\
& =\frac{1}{2^{n}}\left(a_{2}+a_{1}\right)^{n}+\frac{1}{2^{n}}\left(a_{2}-a_{1}\right)^{n} .
\end{aligned}
$$


Next if we calculate $\operatorname{tr}(A+B)^{2}, \operatorname{tr}(A+B)^{3}$, we obtain above equalities similarly as when we determined generators in Section 3 .

Next we consider $\mathbf{C}\left[\mu_{\mathbf{C}}^{-1}(0)\right]^{G L(V, A, \alpha)} \cdot \quad \mathbf{C}\left[\mu_{\mathbf{C}}^{-1}(0)\right]^{G L(V, A, \alpha)}$ is generated by $\{X, Y, Z\}$. The unique relation between $X, Y, Z$ is:

$$
Z^{2}+X^{2} Z+\frac{1}{8} Y^{3}=0
$$

This equation is obtained by the restriction to

$$
\left\{a_{1}=\cdots=a_{6}=0\right\}
$$

the equation which is obtained from the relation in Theorem 4. This equation is a nontrivial quasihomogeneous polynomial with weight $(24: 6,8,12)$. So this equation represents simple singularity of type $E_{6}$ and is irreducible. The equation which is obtained from the relation of Theorem 4 is quasihomogeneous polynomial with weight $(24 ; 2, \ldots, 2,6,8,12)$. Hence the relation which is obtained from the relation of Theorem 4 is irreducible.

4.3. type $\tilde{E}_{7}$. This case is similarly as the case of type $\tilde{E}_{6}$. In this case we have only to calculate $\operatorname{tr}\left(A^{n}\right), \operatorname{tr}\left(B^{n}\right), \operatorname{tr}\left(C^{n}\right), \operatorname{tr}(A+B)^{l}(l=2, \ldots, 7)$.

Next we consider $\mathbf{C}\left[\mu_{\mathbf{C}}^{-1}(0)\right]^{G L(V, A, \alpha)} . \quad \mathbf{C}\left[\mu_{\mathbf{C}}^{-1}(0)\right]^{G L(V, A, \alpha)}$ is generated by $\{X, Y, Z\}$. The unique relation between $X, Y, Z$ is:

$$
Z^{2}+X^{3} Y+Y^{3}=0
$$

This equation is obtained by the restriction to

$$
\left\{a_{1}=\cdots=a_{7}=0\right\}
$$

the equation which is obtained from the relation in Theorem 3. This equation is a quasihomogeneous polynomial with weight $(36 ; 8,12,18)$. So this equation represents simple singularity of type $E_{7}$ and is irreducible. The equation which is obtained from the relation of Theorem 3 is quasihomogeneous polynomial with weight $(36 ; 2, \ldots, 2,8,12,18)$. Hence the relation which is obtained from the relation of Theorem 3 is irreducible.

4.4. type $\tilde{E}_{8}$. This case is similarly as the case of other types. In this case we have only to calculate $\operatorname{tr}\left(A^{n}\right), \operatorname{tr}\left(B^{n}\right), \operatorname{tr}\left(C^{n}\right), \operatorname{tr}(A+B)^{l}(l=2, \ldots, 9)$.

Next we consider $\mathbf{C}\left[\mu_{\mathbf{C}}^{-1}(0)\right]^{G L(V, A, x)}$. $\quad \mathbf{C}\left[\mu_{\mathbf{C}}^{-1}(0)\right]^{G L(V, A, x)}$ is generated by $\{X, Y, Z\}$. The unique relation between $X, Y, Z$ is:

$$
Z^{2}-\frac{1}{8} Y^{3}-\frac{1}{32} X^{5}=0
$$

This equation is obtained by the restriction to

$$
\left\{a_{1}=\cdots=a_{8}=0\right\}
$$

the equation which is obtained from the relation in Theorem 4 . This relation is a quasihomogeneous polynomial with weight $(60 ; 12,20,30)$. So this equation 
represents simple singularity of type $E_{8}$ and is irreducible. The equation which is obtained from the relation of Theorem 4 is quasihomogeneous polynomial with weight $(60 ; 2, \ldots, 2,12,20,30)$. Hence the relation which is obtained from the relation of Theorem 4 is irreducible.

\section{Akashi College of Technology}

\section{References}

[BP] Le Bruyn and C. Procesi, Semisimple representations of quivers, Trans. Amer. Math. Soc., 317-2(1990), 585-598.

[Ki] F. C. Kirwan, Cohomology of quotients in symplectic and algebraic geometry, Math. Notes 31, Princeton univ. Press, (1984).

[Kr] P. B. Kronheimer, The construction of ALE spaces as hyperkahler quotients, J. Differential. Geom., 29-3(1989), 665-683.

[M] J. McKay, Graphs, singularities and finite groups, Proc. Sympos. Pure Math. 37, 183-186.

[N] H. Nakajima, Instantons on ALE spaces, quiver varieties and Kac-Moody algebras, Duke. Math. Journal, 76-2(1994), 365-416.

[Na1] K. Nakamoto, On the structure of the character rings, Master thesis of Kyoto University.

[Na2] K. Nakamoto, The structure of the invariant ring of two matrices of degree 3, preprint.

[S1] P. Slodowy, Simple singularities and simple algebraic groups, Springer Lecture Notes in Math. 815, (1980).

[S2] P. Slodowy, Algebraic Groups and Resolutions of Kleinian Singularities, RIMS-preprint 1086 (1996). 